\title{
Vaccine Literacy
}

\author{
Gauri Kalra ${ }^{1}$, Vijay P Mathur ${ }^{2}$ \\ Journal of South Asian Association of Pediatric Dentistry (2021): 10.5005/jp-journals-10077-3068
}

On January 16, 2021, India began with the world's largest vaccination drive against coronavirus disease-2019 (COVID-19) coronavirus disease-2019 (COVID-19) aiming at immunizing the frontline healthcare workers in the initial phase. The COVID-19 pandemic allowed the country to evaluate its dispensation of effective health care and in doing so; India came out with two major vaccines on emergency use mode, namely, COVISHIELD and COVAXIN. The Government of India has been promoting both vaccines as being equivalent to one another; however, it is believed that Covaxin is being administered as a part of a clinical trial as its efficacy has not been fully established. ${ }^{1,2}$ There are divided opinions about different types of vaccines. As the drive started, hesitation and uncertainty upon vaccine inoculation have been seen with the dental frontline workers too, especially among the younger generation. Such dilemmas may exist due to inadequate knowledge and prevalent rumors regarding the side effects of the new vaccines as there are no long-term efficacy results available for both the vaccines. ${ }^{2}$ The vaccine has now reached almost all the countries in South Asian countries. Thus, vaccine literacy in the present scenario becomes imperative.

\section{How do the Two Vaccine Systems Work?}

COVISHIELD, a recombinant COVID-19 vaccine is based on an Adenovirus vector vaccine system in which a harmless virus transports the virus gene into the human body. The cell then transcribes and translates the gene to produces the antigen (s-protein), which is then displayed on the cell surface to stimulate an immune response. The infected cell may also slowly reproduce the virus which allows more cells to become infected and produce more antigens, thus amplifying the effect. The phase III trials had shown overall efficacy of $70 \%$, ranging from 62 to $90 \%$ with different dosing regimens, with a peer-reviewed safety profile. ${ }^{3}$

COVAXIN is based on a whole-virion inactivated coronavirus vaccine system which includes a whole virus that is killed or inactivated by chemical, heat, or radiation. The vaccine has all the components of the original pathogen to induce a memory response, however, eliminating the possibility of the pathogen replicating and possibly causing infection. Covaxin has also undergone phase III trials and had been approved for restricted emergency use. ${ }^{4}$

\section{Why Two Doses for COVID-19?}

Currently, both vaccines are available for inoculation as $0.5 \mathrm{~mL}$ dose administered intramuscularly as a course of two doses spaced upon the interval of 28 days. Both the vaccines act by stimulating the primary immune response so that the body can develop memory $B$ and T cells against severe acute respiratory syndrome coronavirus 2 (SARS-CoV-2). The development of immune memory by vaccines is what will protect the person against subsequent COVID-19 infection. The body generates two types of immune responses depending upon the number of times the body is exposed to the virus. After the first exposure to the SARS-CoV-2 virus, the primary immune
${ }^{1}$ Department of Pedodontics and Pediatric Dentistry, Sudha Rustagi College of Dental Sciences and Research, Greater Faridabad, Haryana, India

${ }^{2}$ Division of Pedodontics and Preventive Dentistry, Centre for Dental Education and Research, All India Institute of Medical Sciences-WHO Collaborating Centre for Oral Health, New Delhi, India

Corresponding Author: Vijay P Mathur, Division of Pedodontics and Preventive Dentistry, Centre for Dental Education and Research, All India Institute of Medical Sciences-WHO Collaborating Centre for Oral Health, New Delhi, India, Phone: +91 9811955553, e-mail: vijaymathur7@gmail.com

How to cite this article: Kalra G, Mathur VP. Vaccine Literacy. J South Asian Assoc Pediatr Dent 2021;4(1):1-2.

Source of support: Nil

Conflict of interest: None

response is slow and weak as it takes days for the body to generate enough antibodies and T cells to eliminate the virus. However, longlasting memory B and T cells that "remember" the SARS-CoV-2 virus are produced generating immune memory. A secondary immune response is generated by the body when the virus enters the body for the second time. This secondary response is stronger and quicker than the primary immune response as memory $B$ and $T$ cells are rapidly activated resulting in higher antibody concentrations and $T$ cell counts around the body to eliminate the virus more quickly, reducing the symptoms and severity of COVID-19. ${ }^{5}$ The researchers are still debating on possibility of increasing the gap between two vaccine doses to enhance body immune response.

\section{Who Can Take the COVID-19 Vaccines?}

All individuals attaining the age of 18 years are eligible to take the vaccine except pregnant and lactating females as the trials had not been conducted yet including them. History of anaphylaxis (immediate or delayed) to the previous dose of COVID vaccine or known allergies to injectable vaccines or certain therapies, pharmaceutical products, food items are contraindicating conditions for COVID vaccine administration. But, temporary contraindications may include persons with active SARS-CoV-2 infection, those given anti-SARS-CoV-2 monoclonal antibodies, and plasma and extremely unwell patients with other chronic ailments. Moreover, special precautions must be taken in persons with a known history of bleeding disorders, platelet disorders, clotting factor disorder, and coagulopathies. Importantly, there is no contraindication for individuals with chronic morbidities such as cardiac, neurological, pulmonary, metabolic, malignancies, etc.; patients with a history of SARS-CoV-2 infection and with HIV, immune-suppression, however, the effect of COVID vaccine may be slightly reduced in such cases. COVID vaccine administration must be separated with a gap of 2 weeks from any other vaccine 
in all the individuals. The interchangeability of type of vaccine is not acceptable which means that the second dose should be of the same vaccine which was administered for the first dose. ${ }^{6}$

\section{Are the COVid-19 Vaccine Systems SAFE?}

Vaccine development undergoes several clinical trials before initiating its mass use. In phase I of the trials, any new vaccine is assessed for any risk factors or adverse effects, the dose required, whether this dose is the same for different individuals, and if the vaccine promotes healthy immune systems to make antibodies. If no risk factors or adverse effects are found in phase I trials, then, phase II and phase III trials expand to involve more volunteers, increasing statistical power. FDA approval before licensing and distribution is mandatory after phase III trials. However, phase IV trials being the last phase includes ongoing studies of risk and side effects after the vaccine is distributed. Phase III trials of both vaccine systems have been proven to be safe with some mild AEFI such as injection site tenderness, injection site pain, headache, fatigue, myalgia, malaise, pyrexia, chills, arthralgia, and nausea. Dizziness-giddiness, tremor, sweating, cold, and cough may be associated additionally with COVAXIN. Paracetamol and levocetirizine may be used to provide symptomatic relief from postvaccination adverse reactions with COVISHIELD. Very rare events of demyelinating disorders have been reported following vaccination with this vaccine without the causal relationship establishment. As with other intramuscular injections, COVISHIELD should be given with caution to individuals with thrombocytopenia. Special indications with COVAXIN include being shaken well before use and the use of chloroquine and corticosteroids is not advised to be used along with vaccination as that may impair antibody response. ${ }^{7}$
Last year had taught us to accept the new normal of surviving with SARS-CoV-2 during the pandemic, however, the New Year brings hope and positivity with the introduction of large scale antiSARS-CoV-2 vaccine drive throughout the country which assures to end the pandemic soon. Hence, dilemmas regarding the use of COVID-19 vaccines should not exist.

\section{References}

1. Molaei S, Dadkhah M, Asghariazar V, et al. The immune response and immune evasion characteristics in SARS-CoV, MERS-CoV, and SARSCoV-2: Vaccine design strategies. Int Immunopharma 2020;92:107051. DOI: 10.1016/j.intimp.2020.107051.

2. Hodgson SH, Mansatta K, Mallett G, et al. What defines an efficacious COVID-19 vaccine? A review of the challenges assessing the clinical efficacy of vaccines against SARS-CoV-2. Lancet Infect Dis 2020;21(2):e26-e35. DOI: 10.1016/S1473-3099(20)30773-8.

3. Voysey M, Clemens SA, Madhi SA, et al. Safety and efficacy of the ChAdOx1 $\mathrm{nCoV}-19$ vaccine (AZD1222) against SARS-CoV-2: an interim analysis of four randomised controlled trials in Brazil, South Africa, and the UK. The Lancet 2020;397(10269):99-111. DOI: 10.1016/S01406736(20)32661-1.

4. Bharat Biotech Recruits 23,000 Volunteers ForCovaxin's Phase 3 Clinical Trial". NDTV.com. 28 December 2020. Retrieved 3 January 2021.

5. Lin DY, Zeng D, Mehrotra DV, et al. Evaluating the efficacy of COVID-19 vaccines. Clin Infect Dis 2020. ciaa1863. DOI: 10.1093/cid/ ciaa1863.

6. Emadi A, Chua JV, Talwani R, et al. Safety and efficacy of imatinib for hospitalized adults with COVID-19: a structured summary of a study protocol for a randomised controlled trial. Trials 2020;21(1):897. DOI: 10.1186/s13063-020-04819-9.

7. Lipsitch M, Dean NE. Understanding COVID-19 vaccine efficacy. Science 2020;370(6518):763-765. DOI: 10.1126/science. abe5938.

Important Notice

The authors shall get the ORCID credits for having publication in this journal.

The Journal is now indexed with DRJI, I2OR, J Gate and Index Copernicus.

The Journal Manuscript by authors is now being given credits as National Professional Journal and is accessible through Dental Council of India centralized Journal access for dental institutions under Jaypee Journals. 